\title{
A Methodology for Detection and Estimation of Software Aging
}

\author{
Sachin Garg, Aad van Moorsel \\ Lucent Technologies \\ Bell Laboratories \\ 600 Mountain Avenue \\ Murray Hill, NJ 07974, USA \\ \{sgarg,aad\}@ research.bell-labs.com
}

\author{
Kalyanaraman Vaidyanathan, Kishor S. Trivedi \\ Center for Advanced Computing \& Communication ${ }^{\dagger}$ \\ Dept. of Electrical \& Computer Engineering \\ Duke University \\ Durham, NC 27708, USA \\ $\{k v, k s t\} @ e e . d u k e . e d u$
}

\begin{abstract}
The phenomenon of software aging refers to the accumulation of errors during the execution of the software which eventually results in it's crash/hang failure. A gradual performance degradation may also accompany software aging. Pro-active fault management techniques such as "Software rejuvenation" [9] may be used to counteract aging if it exists. In this paper, we propose a methodology for detection and estimation of aging in the UNIX operating system. First, we present the design and implementation of an SNMP based, distributed monitoring tool used to collect operating system resource usage and system activity data at regular intervals, from networked UNIX workstations. Statistical trend detection techniques are applied to this data to detect/validate the existence of aging. For quantifying the effect of aging in operating system resources, we propose a metric "Estimated time to exhaustion" which is calculated using well known slope estimation techniques. Although the distributed data collection tool is specific to UNIX, the statistical techniques can be used for detection and estimation of aging in other software as well.
\end{abstract}

\section{Introduction}

It is now well established that outages in computer systems are more due to software faults than due to hardware faults $[6,19]$. Recently, the phenomenon of software aging [9] has come to light, where the error conditions actually accrue with time and/or load, resulting in performance degradation and/or failures. Failures of both crash/hang type as well as those resulting in data inconsistency have been reported. Memory bloating and leaking, unreleased

\footnotetext{
* This work was supported in part by an IBM Fellowship to CACC Duke University and was partly performed while the author was at Duke

${ }^{\dagger}$ This work was supported in part by a core project of the CACC, Duke University
}

file-locks, data corruption, storage space fragmentation and accumulation of round-off errors are some typical causes of slow degradation. Not only software used on a mass scale (most PC users are familiar with applications that occasionally "hang"), but also specialized software used in high-availability and safety-critical applications suffers from aging [9]. To counteract aging, a pro-active approach to environment diversity has been proposed in which the operational software is occasionally stopped and then restarted in a "clean" internal state. Huang et. al. [9] have proposed a technique called "software rejuvenation," which involves stopping the running software occasionally, removing the accrued error conditions and restarting the software. Some examples of cleaning the internal state of software are garbage collection, flushing operating system kernel tables and reinitializing internal data structures. An extreme example of rejuvenation might be a simple hardware reboot.

The existence of software aging has been widely reported and observed, but has been mainly anecdotal. For this reason, in this paper, we describe a methodology based on well known statistical techniques for detection and validation of the existence of software aging. It is clear that the study of aging cannot be based on traditional measurement techniques for dependability assessment, which rely on data from failure events. The basic idea is to periodically monitor and collect data on the attributes responsible for determining the "health" of the executing software. Statistical trend detection techniques are applied to this data to detect and validate the existence of aging. In this paper, we demonstrate this simple methodology for the UNIX operating system. First, we present the design and implementation of a distributed monitoring tool used to collect operating system resource usage and system activity data at regular intervals, from networked UNIX workstations. This tool is based on the Simple Network Management Protocol (SNMP) standard simply to be able 
to inter-operate with multiple platforms. For quantifying the effect of aging in operating system resources, we propose a metric "Estimated time to exhaustion" which is calculated using well known slope estimation techniques. Although the distributed data collection tool is specific to UNIX, the statistical techniques can be used for detection and estimation of aging in other software as well.

The rest of this paper is organized as follows. In Section 2, we give a brief overview of previous work in measurement-based software dependability evaluation and explain some differences between that and our work. Description of the SNMP framework and the distributed resource monitoring tool is given in Section 3. In Section 4, we describe the experimental set up for collecting data. Section 5 contains an overview of the statistical methods employed to analyze the collected data. The experimental measurement and analysis results are discussed in Section 6.

\section{Related Work}

Most of the previous work in measurement-based dependability evaluation was based on measurements made at either failure times $[3,10,14]$ or at times an error was observed [11, 15, 20, 21]. Chillarege et. al. [3] gave a first order empirical estimation of the failure rate and mean time to failure of widely distributed software. The effect of system workload on system failures was investigated in [10]. Iyer et. al. [11] proposed a methodology for recognizing the symptoms of a persistent problem in large systems by identifying and statistically validating recurring patterns among error records produced in the system. Hansen and Siewiorek [7] proposed a technique for coalescing error events for data reduction in the case of multiple errors due to a single fault. The dispersion frame technique for failure prediction, based on an increase in observed error rate, a threshold error number, a CPU utilization threshold or a combination of the above factors, was described in [15].

Since software aging cannot be detected or estimated by collecting data at failure events only, we, by contrast, periodically monitor the behavior of software in operation. Also, while some the above papers dealt with hardware failures, we restrict ourselves to software failures.

Constant monitoring of system parameters was carried out by Maxion and Feather [16], who described a method to automatically detect anomalies in the system behavior. While their focus was on the network (Ethernet), we aim to detect aging and other anomalies in UNIX workstations. They applied smoothing techniques like median filtering and thresholding in their analysis and aimed to diagnose the original problem caused by the observed anomaly. Our analysis of trend detection and estimation (part of which includes non-parametric local regression for smoothing), is aimed towards validation of software aging and estimation of time to exhaustion of various operating system resources any of which will result in a failure.

\section{SNMP-based Distributed Resource Moni- toring Tool}

Simple Network Management Protocol [2, 17] is an application protocol offering network management services in the Internet protocol suite. There are three essential constituents to a management tool based on SNMP: the manager, the agent and the Management Information Base (MIB). A client-server relationship between manager and agent is defined by the SNMP protocol and the MIB describes the information that can be obtained and/or modified via interactions between the agent and the manager.

We have used the SNMP framework to design and implement a distributed resource monitoring tool. The objective of this tool is to remotely monitor the health of UNIX workstations at the operating system level. The data collected while monitoring is then subjected to statistical analysis. The three key components of the tool are:

1. PFM MIB: The Pro-active Fault Management (PFM) MIB module defines the objects used to determine the "health" of a UNIX workstation. Figure 1 shows the MIB tree for the PFM module. Note that objects for proprietary MIBs are defined under an organization's subtree located under the "enterprises" branch. The module "pfmMIB" is defined as \{enterprises $2598\}$ in the MIB tree hierarchy, where the number 2598 is arbitrarily given. The objects to be monitored are classified in the following seven categories, each of which is defined as a placeholder object in the module.

(a) hostID defined as \{pfmMIB 1\}: The leaf objects in this category, nodeName, osName, osRelease, osVersion and mcHardwareName, characterize the features of the workstation. Since the value of each of these objects is constant, they need to be retrieved by the remote manager/monitor only once.

(b) timeVal defined as $\{$ pfmMIB 7$\}$ : It contains two leaf objects, dateAndTime, which is the current day and time, and hostUpstats, which is the time the machine has been up since last reboot. 


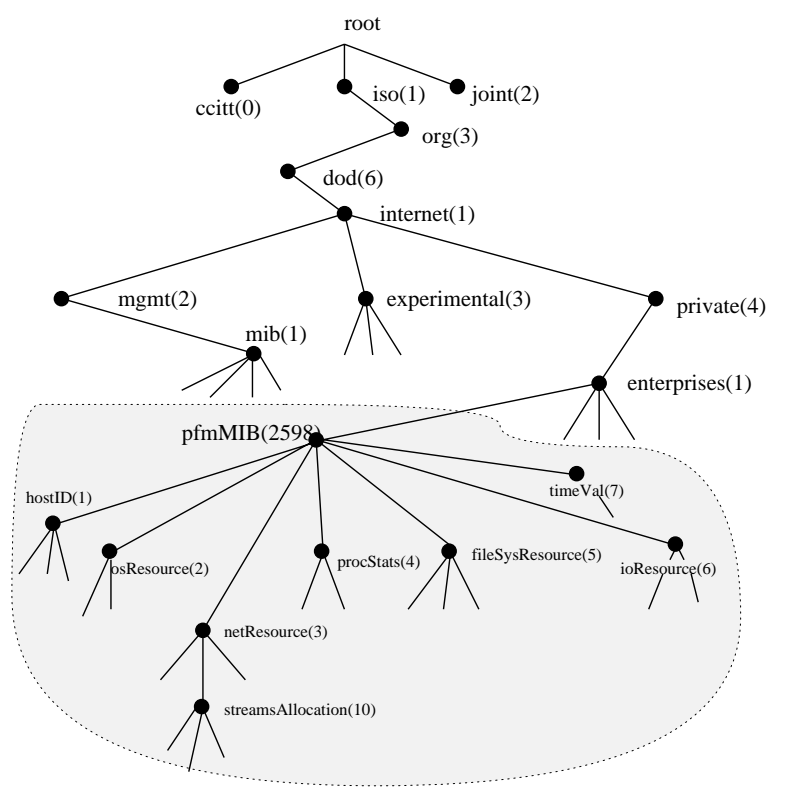

Figure 1. MIB tree for the PFM module

(c) osResource defined as \{pfmMIB 2\}: The leaf objects here describe the state of the resources provided by the OS and so some of the objects are operating system dependent. Some of the objects are usedSwapspace, fileTableSize, realMemoryFree and procstotal.

(d) procstats defined as \{pfmMIB 4\}: It contains leaf objects that describe the state of the processes running on the machine.

(e) filesysResource defined as \{pfmMIB 5\}: It consists of four leaf objects tmpDirsize, tmpDirUsed, tmpDirAvail and tmpDirCapacity, which keep track of the /tmp directory in UNIX systems.

(f) netResource defined as \{pfmMIB 3$\}$ : It is a placeholder object in PFM MIB to monitor the availability and usage of network related resources provided by the operating system. Some examples are queuescurrent, dblksAllocFail and streamsCurrent.

(g) ioResource is defined as \{pfmMIB 6\}: It contains information about the terminal as well as disk I/O activity such as ttyIn and diskOneMsps. A total of fourteen leaf objects comprise this category.

2. PFM agent: The agent process runs in the background on each monitored workstation. A single instance for each of the MIB leaf objects is initialized as a global variable in the agent program. The agent then passively listens on a well advertised port number. Upon receiving a get request from the manager, the agent executes certain instructions to obtain the value of the requested leaf object from the operating system, assigns the value to the variable and sends it back to the manager with the value. This procedure is illustrated in Figure 2. The values for the leaf objects are obtained by the agent by executing various UNIX utility programs made available as a standard part of the operating system such as pstat, iostat, netstat, vmstat, nfsstat, top and df.

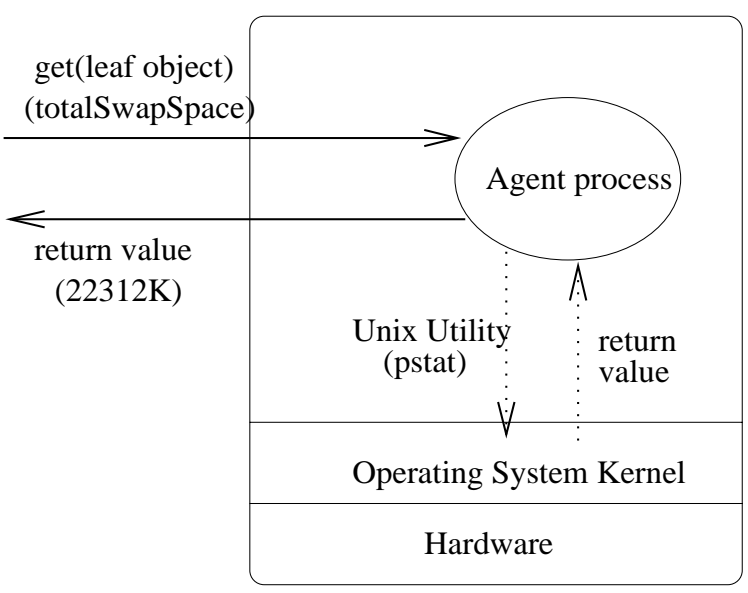

Figure 2. Working of the agent process

3. PFM Manager: The manager's primary function is to retrieve the values of desired objects by sending get requests to the remote agents. We have implemented a graphical user interface which can be operated in one of two modes - "monitoring" and "collection". While in the monitoring mode, the retrieval of information is initiated by the user (administrator), in the collection mode, it is automated. Figure 3 shows the snapshot of the interface with some selected objects and their associated values.

The prototypes for the agent and the manager programs were developed using Scotty [13]. Scotty is an extension to the Tcl/Tk prototyping language and provides TCP/IP, UDP, ICMP and SNMP functionality. It supports both versions 1 and 2 of SNMP. In our implementation, we have used only SNMP V.1.

\section{Data Collection}

The SNMP-based data collection tool described in the previous section was deployed on eight heterogeneous 


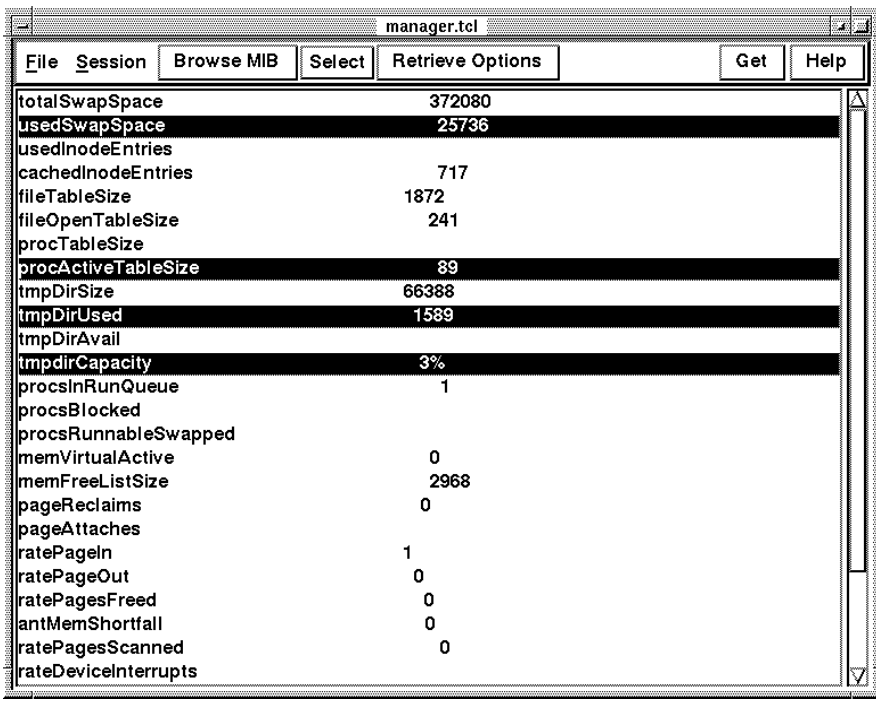

Figure 3. Snapshot of the GUI for the manager

UNIX workstations which are connected by an Ethernet LAN at the Duke Department of Electrical and Computer Engineering. The machines monitored and their respective operating systems and primary functions in the department are listed in Table 1. In our data collection, the values for all objects are collected every fifteen minutes using the collection mode. The data is stored in a user-specified file in an $\mathrm{X}-\mathrm{Y}$ format, where $\mathrm{X}$ is the name of the object and $\mathrm{Y}$ is its value. Any processing on the data is done outside of the tool. In the case of an error or timeout, a "No Response" is recorded. The timeout interval was two minutes. When a monitored machine fails, it is restarted/rebooted and the agent process is also restarted.

\section{Data Analysis}

Using the monitoring tool, time ordered values are obtained for each pfmMIB object, thus constituting a time series for that object. In this section, we introduce the time series analysis concepts used to determine the patterns of variation with time in the values of each of the objects. Specific issues we address are:

- Is aging present, or in other words, is there a long term trend (increasing or decreasing) in the values?

- What is the nature of the variations in values? Does the data exhibit periodic behavior with or without a global/local trend?

- Can the failures during this period be related to the observed values?
Table 1. Machines monitored at Duke Dept. of ECE

\begin{tabular}{|c|c|c|}
\hline "Machine Name & Operating System & Function \\
\hline ECE & Solaris 2.2 & $\begin{array}{l}\text { WWW, FTP, mail, } \\
\text { NIS. }\end{array}$ \\
\hline Washington & Solaris 2.3 & $\begin{array}{l}\text { file server } \\
\text { for programs/packages }\end{array}$ \\
\hline Lincoln & Solaris 2.2 & $\begin{array}{l}\text { file server for directories, } \\
\text { research printer server }\end{array}$ \\
\hline Jefferson & SunOS 4.1.3 & NIS secondary server \\
\hline Dolphin & SunOS 4.1.3 & $\begin{array}{l}\text { research (usr home } \\
\text { directories) }\end{array}$ \\
\hline Datc6 & Solaris 2.2 & public cluster workstation \\
\hline Velum & SunOS 4.1.3 & $\begin{array}{l}\text { research (usr home } \\
\text { directories) }\end{array}$ \\
\hline Rossby & SunOS 4.1.3 & research \\
\hline Shannon & AIX & $\begin{array}{l}\text { research, data collection } \\
\text { /monitoring station }\end{array}$ \\
\hline
\end{tabular}

- Can we quantify aging in UNIX?

To obtain the desired answers, we use visual cues and classical time series analysis techniques such as linear and periodic dependency analysis, and trend detection and estimation [1].

\subsection{Time Series Analysis and Time Plots}

A time series is simply a set of data $\left\{y_{t}: t=1, \ldots, n\right\}$ in which the subscript $t$ indicates the time at which the datum $y_{t}$ is observed. It is a sample realization of a stochastic process $\left\{Y_{t}: t=1, \ldots, n\right\}$. The expectation of $Y_{t}$, denoted by $\mu_{t}=E\left[Y_{t}\right]$ is called the trend of the series. In our case, since the observations are made at regular fifteen minute intervals, it suffices to indicate the index of the interval.

Plotting the time series $\left\{y_{t}: t=1, \ldots, n\right\}$, against time $t=1, \ldots, n$ is the first step in data analysis. The visual cues are helpful in determining further analysis to be performed. For instance, periodicities may be clearly seen, indicating the need for harmonic analysis, or a trend (increasing or decreasing) may be visible, indicating that trend estimation techniques may be applied. Further, if both are visible, then it is at times necessary to use statistical methods to remove the trend in order to perform harmonic analysis. On the other hand, it is sometimes desirable to remove any cyclic variations and just study the trend, particularly for 
forecasting purposes.

\subsection{Periodicity and Linear Dependence}

To study linear dependency in time, the autocorrelation function is an important, albeit typically incomplete, summary of the serial dependence within a stationary random function. The plot of autocorrelation against lag, called the correlogram of the data, is very useful for exploratory data analysis. In addition to linear dependence, periodic dependencies may be important. To corroborate the presence of periodicities in the data, we employ harmonic analysis, which involves fitting a periodic function of a particular frequency to the data. The existence of daily and weekly periodicities in the measured data can be confirmed via harmonic analysis.

\subsection{Trend Detection and Estimation}

As mentioned previously, one of the primary objectives of our data analysis is to detect and validate the existence of aging. Detection of trends in operating system resource usage and system activity is the approach we follow. For the purposes of prediction, the slope of the trend is estimated. The primary trend detection technique used is smoothing of observed data by robust locally weighted regression, proposed by Cleveland [4]. The process of robust locally weighted regression essentially involves fitting a polynomial of any desired degree to a fraction of the data. The fitting is carried out using weighted least squares estimates. The fraction of the data smoothed at each point, called the window size, has a significant effect on the result. A large window size is used to capture the overall trend by removing local variations. With a small window size, the smoothed data points almost follow the original data points. We have used a window size of $2 / 3$ (fraction of all data) for our analysis. The robust smoothing technique provides good visual cues for finding trends in the series $y_{t}$, but it is hard to make conclusive statements regarding presence or absence of trend since no statistical significance levels are attached. To overcome this limitation, we use the seasonal Kendall test [5]. It can be used for detection of trends in the presence of cycles. The duration of a cycle is referred to as the period and the durations within a cycle are called seasons. We expect to see daily cycles in our data. The objective in the seasonal Kendall test is to test the null hypothesis $H_{0}$ that there is no trend, against the alternative hypothesis $H_{A}$ that there is an upward or a downward trend. To that end, we compute for each season $i$ the Mann-Kendall statistic, $S_{i}$, where

$$
S_{i}=\sum_{k=1}^{n_{i}-1} \sum_{l=k+1}^{n_{i}} \operatorname{sgn}\left(y_{i l}-y_{i k}\right),
$$

where $l>k$ and $\operatorname{sgn}(x)$ is the signum function, $n_{i}$ is the number of data (over cycles) for season $\mathrm{i}$ and $y_{i l}$ is the datum for the $i$ th season of the $l$ th cycle. To test the null hypothesis, $H_{0}$ is rejected for certain values of the $Z$ statistic (computed from all the $S_{i} \mathrm{~s}$ ), for a significance level $\alpha$ (see [5] for details). The seasonal Kendall test is simple, efficient and robust against any missing values in the data.

Once the presence of a trend is confirmed by the above procedure, its true slope may be estimated by computing the least squares estimate of the slope by linear regression methods. These however deviate greatly from the true value if there are gross errors or outliers in the data. We use a non-parametric procedure developed by Sen [18], which is not greatly affected by outliers and is also robust against missing values.

First, for each season $i, N_{i}^{\prime}$ slopes are calculated for all pairs of points at $l$ and $k$ for which $l>k$, as $Q_{i}=\left(y_{i l}-y_{i k}\right) /(l-k)$. These $N_{1}^{\prime}+\ldots+N_{K}^{\prime}=N^{\prime}$ slopes, where $K$ is the number of seasons, are then ranked and their median is calculated. This median is the required slope estimate, $N$. A confidence interval can also be attached to the slope.

\section{Results}

The total time period for our data collection was approximately 53 days. Three machines, Jefferson, Rossby and Velum, did not experience any outage during this period. The other four machines, Dolphin, ECE, Lincoln and Datc6, suffered outages. The number of outages, the time of occurrence of each outage and its probable cause are listed in Table 2. The time of outage is expressed in days:hours:minutes. The probable cause of failure is noted simply by observing the values of the objects just before the failure and identifying resources, if any, that are nearing exhaustion at that time. Failures which did not show any signs of resource exhaustion (presumably due to hardware or other faults) are not considered in our analysis.

The time plots for the monitored objects have missing values if there is an outage or in the case of monitor timeouts occurring on get requests. Figure 5 illustrates the four outages in Datc6, and Figure 6 and 7 show the outage in ECE. We will get back to these figures when discussing 
Table 2. Observed number, times and probable causes of failures

\begin{tabular}{|c|c|c|c|}
\hline \hline Machine & Outages & Time & Cause \\
\hline \hline Dolphin & 3 & $01: 21: 55$ & Swap Space Exhausted \\
& & $20: 16: 45$ & \\
& & $50: 09: 51$ & \\
\hline ECE & 1 & $16: 17: 34$ & Out of Memory \\
\hline Lincoln & 4 & $12: 08: 03$ & Out of Memory \\
& & $30: 03: 55$ & Too many messages \\
& & $38: 10: 13$ & \\
& & $45: 12: 49$ & \\
\hline Datc6 & 4 & $12: 08: 09$ & Outages \\
& & $30: 09: 47$ & Correlated \\
& & $38: 11: 03$ & with \\
& & $45: 13: 44$ & Lincoln \\
\hline \hline
\end{tabular}

Series : Current dblks
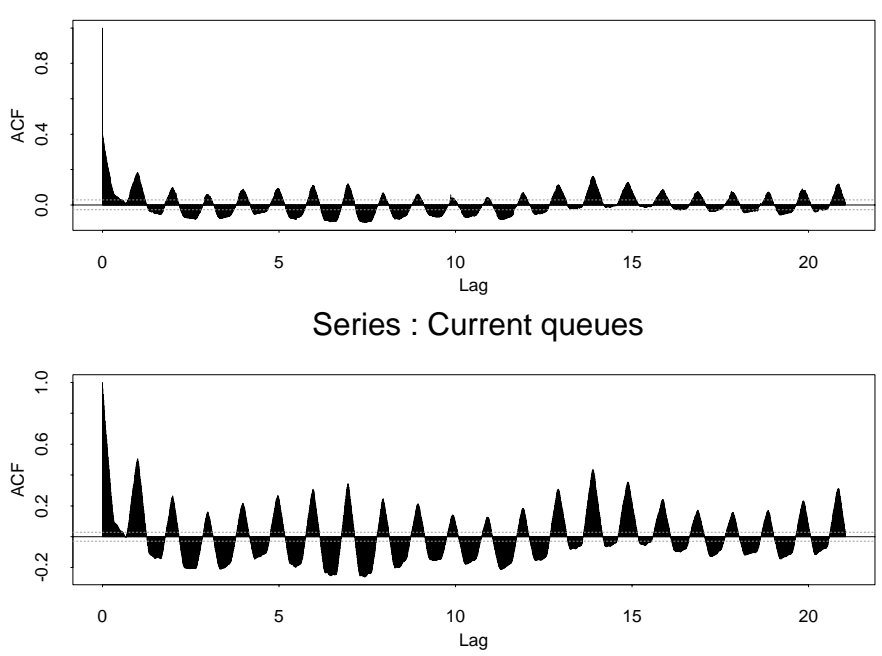

Figure 4. Autocorrelation function for Jefferson objects: (1) current no. of allocated streams data blocks (2) no. of stream queues
Time plots for Datc6 objects
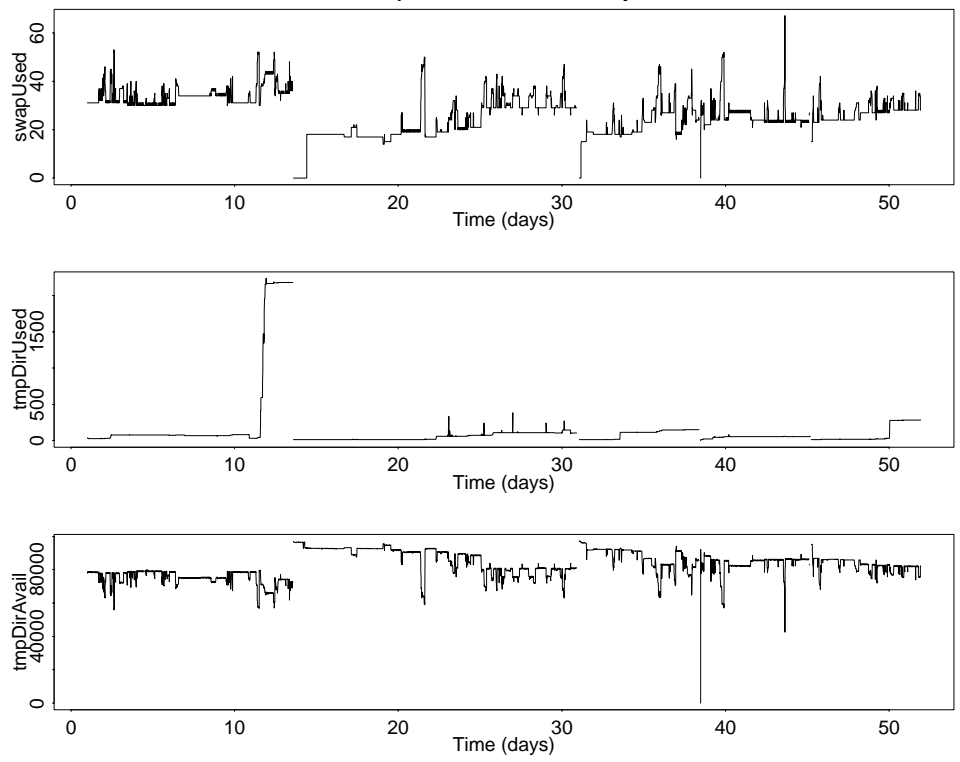

Figure 5. Time plots for Datc6 objects: (1) swap space in use (2) /tmp in use (3) /tmp available
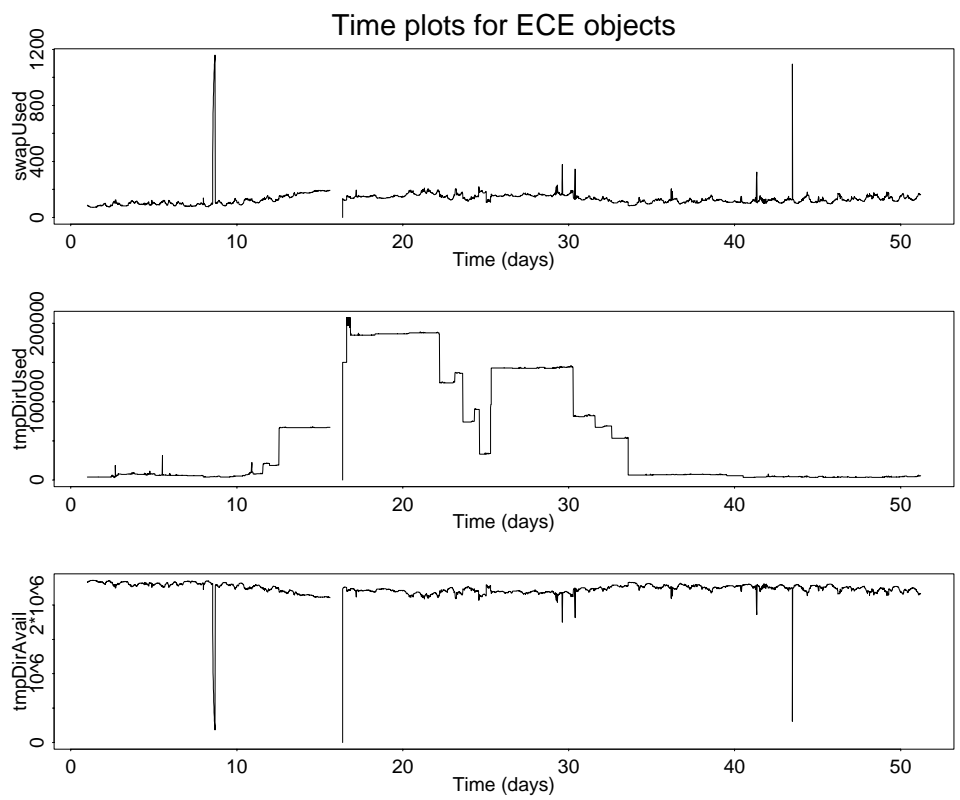

Figure 6. Time plots for ECE objects: (1) swap space in use (2)/tmp in use (3) /tmp available 

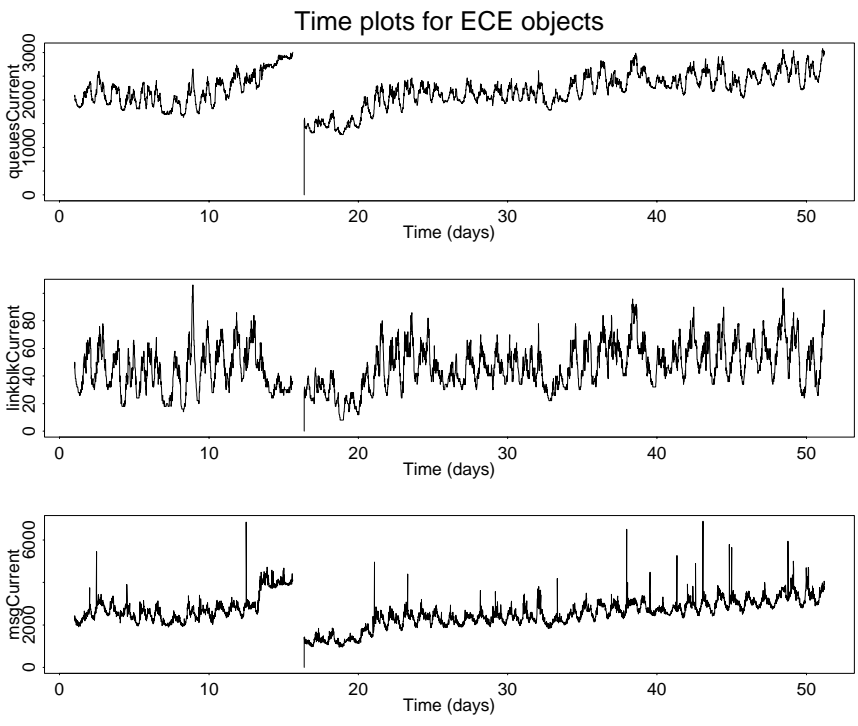

Figure 7. Time plots for ECE objects: (1) current no. of stream queues (2) linkblk STREAMS structures allocated (to indicate a link) (3) streams messages allocated

trends. First, we identify linear and periodic dependencies.

Figure 4 shows the autocorrelation functions plotted against lag for two objects monitored in machine Jefferson. Computing the autocorrelation function requires that the time series $y_{t}$ be stationary. However, the time series potentially consist of a global trend, which makes them non-stationary. Therefore, this trend, $\mu_{t}$ is computed using non-parametric regression smoothing and subtracted from the original time series $y_{t}$ and the autocorrelation functions are computed for these residual series $y_{t}-\mu_{t}$ for lags of up to approximately 20 days. Hence the lag in the plots corresponds to days. Each plot contains two horizontal dashed lines which correspond to $95 \%$ confidence limits. Statistically, correlation functions lying inside these boundaries are considered insignificant.

\subsection{Detection of Periodicities and Linear Depen- dence}

Figure 4, plots 1 and 2, show significant autocorrelation at the lag which corresponds to a day. As the autocorrelation persists over all lags (visible as alternating positive and negative peaks), it is a clear indication that the time series has a periodic component with a periodicity of one day. Furthermore, the envelope of the peaks in these plots show that correlations exist at a lag of 7, which corresponds to a week. This is indicative of the presence of weekly periodicity exhibited by the original time series.

Analysis of periodic dependencies can be done for all objects. Not all objects show daily and weekly dependencies as in plots 1 and 2 of Figure 4. Some only show weekly dependencies (for instance caused by periodic maintenance activities), while others show no significant indications of the presence of periodicities or linear dependence.

\subsection{Detection and Validation of the Existence of Aging}

The existence of aging is some times evident simply from observation of some of the time plots of resources. For instance, a gradual consistent increase in resource usage (disregarding local variations) is visible for some of the ECE objects in Figures 6 and 7. In the case of other resources, however, aging is not evident simply by visual inspection of data. Hence, we resort to the analysis techniques described earlier to detect aging.

Smoothing of a time series by non-parametric local weighted regression is applied to the time series of objects for Rossby. Figure 8 shows the smoothed data superimposed on the original data points. Amount of real memory free, plotted in Figure 8, plot 1, shows an overall decrease, whereas process table size shows an increase. We have used the smoothing technique only to get the global trend between outages and so the resulting smoothed data might not always follow the original data points. Plots of some other resources not discussed here also showed an increase or decrease. Once again, this corroborates our hypothesis of aging with respect to various objects.

We also applied the seasonal Kendall test to each of these time series to detect the presence of any global trends at a significance level, $\alpha$, of 0.05 . The associated statistic is listed in Table 3. With $Z_{\alpha}=1.96$, all values in the table are such that the $H_{0}$ hypothesis (that no trend exists) is rejected.

The above trend analysis lends itself to failure prediction (as we will see later in this section), but does not explicitly deal with periodicity. Therefore, we show in Figure 9 the seasonal trend decomposition of the time series of file table size on Jefferson plotted against time in weeks. The topmost plot in Figure 9 is the original time series. The second one shows the global trend of increase in the size of the file table. It is obtained by non-parametric regression. The third plot from the top shows the periodicities, in which the daily cycle is a harmonic of the weekly cycle. The bottom plot shows the remainder. Summation of corresponding 

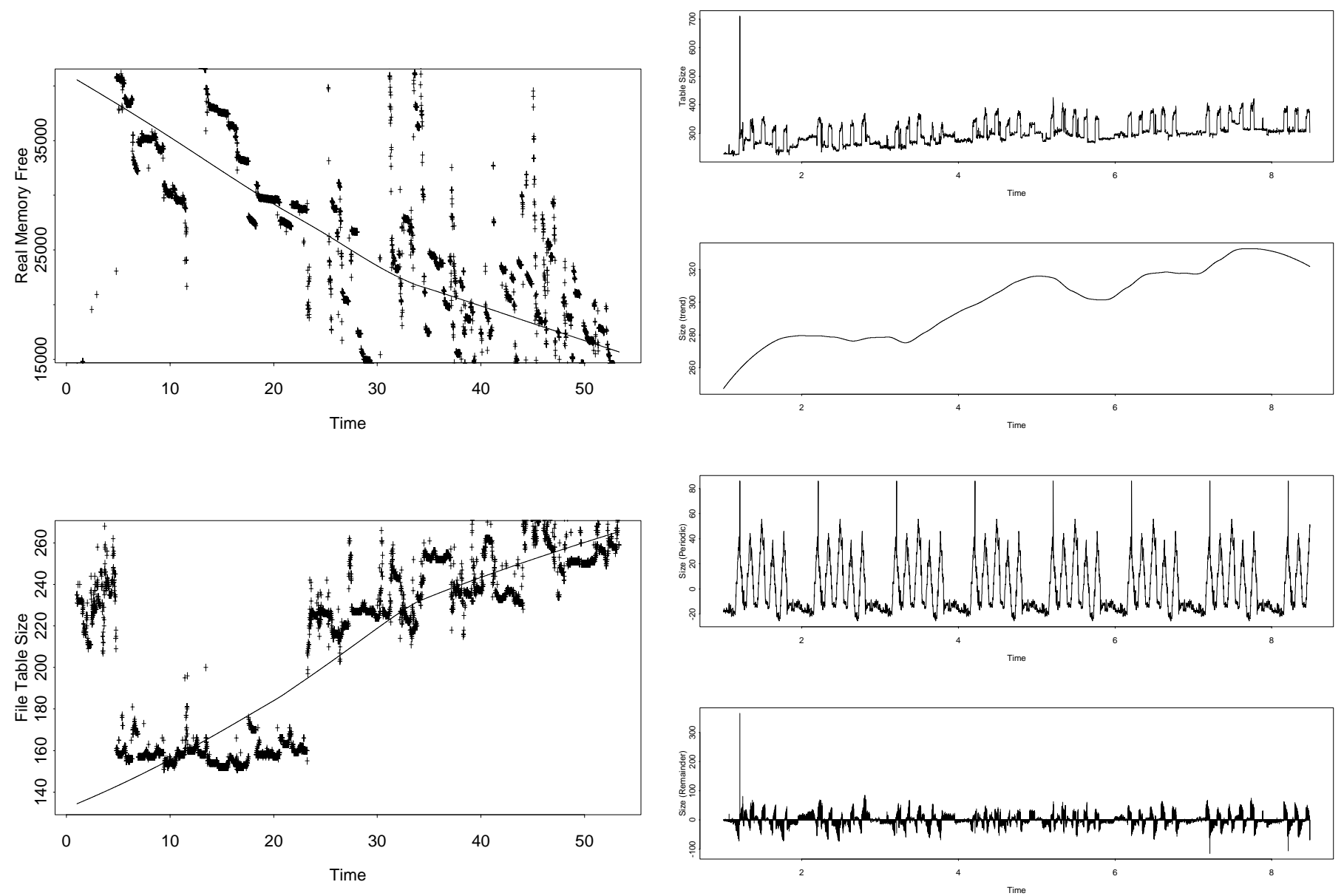

Figure 8. Non-parametric regression smoothing for Rossby objects: (1) free memory and (2) file table size

\section{Figure 9. Trend and seasonal decomposition for Jefferson object file table size against time in weeks}

Table 3. Seasonal Kendall test (Z Statistic) for Rossby, Velum and Jefferson objects at $\alpha=0.05$

\begin{tabular}{|c|c|c|c|}
\hline \hline $\begin{array}{c}\text { Resource } \\
\text { Name }\end{array}$ & Rossby & Velum & Jefferson \\
\hline \hline Real Memory Free & -13.668 & -6.848 & -46.977 \\
File Table Size & 38.001 & 17.006 & 47.065 \\
Process Table Size & 40.540 & 12.142 & 38.537 \\
Used Swap Space & 15.280 & 32.654 & 31.660 \\
No. of disk data blocks & 48.840 & 13.955 & 13.673 \\
No. of queues & 39.645 & 19.906 & 13.476 \\
\hline \hline
\end{tabular}

$Y$ values of the bottom three plots yields the original time series. The difference in scale between the three plots is worth noting as it gives the relative significance of the three components. Particularly noteworthy is the sharp spike present in the original time series as well as the remainder component at around the 2nd day of the first week. This remains unexplained and is attributed to random phenomenon. Such extremely significant yet unexplained transients are present in other time series objects as well.

\subsection{Age Quantification and Estimation}

Given that a global trend is present and that its slope is calculated for a particular resource, we estimate the time at which the resource will be exhausted because of 
Table 4. Estimated slope and time to exhaustion for Rossby, Velum and Jefferson objects

\begin{tabular}{|c|c|c|c|c|c|}
\hline \hline $\begin{array}{c}\text { Resource } \\
\text { Name }\end{array}$ & $\begin{array}{c}\text { Initial } \\
\text { Value }\end{array}$ & $\begin{array}{c}\text { Max } \\
\text { Value }\end{array}$ & $\begin{array}{c}\text { Sen's Slope } \\
\text { Estimation }\end{array}$ & $\begin{array}{c}\text { 95\% Confidence } \\
\text { Interval }\end{array}$ & $\begin{array}{c}\text { Estimated Time } \\
\text { to Exhaustion (days) }\end{array}$ \\
\hline \hline Rossby & & & & & \\
Real Memory Free & 40814.17 & 84980 & -252.00 & $-287.75:-219.34$ & 161.96 \\
File Table Size & 220 & 7110 & 1.33 & $1.30: 1.39$ & 5167.50 \\
Process Table Size & 57 & 2058 & 0.43 & $0.41: 0.45$ & 4602.30 \\
Used Swap Space & 39372 & 312724 & 267.08 & $220.09: 295.50$ & 1023.50 \\
Velum & & & & & \\
Real Memory Free & 63276.03 & 116924 & -188.00 & $-253.91:-132.31$ & 336.57 \\
File Table Size & 251 & 3628 & 0.67 & $0.58: 0.70$ & 5065.50 \\
Process Table Size & 60 & 1034 & 0.16 & $0.13: 0.17$ & 6168.67 \\
Used Swap Space & 17516.01 & 262076 & 418.00 & $394.22: 446.00$ & 585.07 \\
Jefferson & & & & & \\
Real Memory Free & 67638.54 & 114608 & -972.00 & $-1006.81:-939.08$ & 69.59 \\
File Table Size & 268.83 & 7110 & 1.33 & $1.30: 1.38$ & 5144.36 \\
Process Table Size & 67.18 & 2058 & 0.30 & $0.29: 0.31$ & 6696.41 \\
Used Swap Space & 47148.02 & 524156 & 577.44 & $545.69: 603.14$ & 826.07 \\
\hline \hline
\end{tabular}

aging only. Table 4 refers to several objects on Rossby, Velum and Jefferson and lists an estimate of the slope (change per day) of the trend obtained by applying Sen's non-parametric method. The values for real memory and swap space are in Kilobytes. A negative slope, as in the case of real memory, indicates a decreasing trend, whereas a positive slope, as in the case of file table size, is indicative of an increasing trend. Given the slope estimate, the table lists the estimated time to failure of the machine due to aging only with respect to this particular resource. The calculation of the time to exhaustion is done by using the initial intercept, $c$, the calculated slope, $m$, and a standard linear approximation $y=m x+c$. The value of the intercept $c$ is taken to be the mean of the initial 5 days. The minimum value for all the resources is zero.

A comparative effect of aging on different system resources can be obtained from the above estimates. For example, in machine Rossby, the resource used swap space has the highest slope and real memory free has the second highest slope. Therefore, used swap space has the highest rate of exhaustion. However, when we compare the estimated times to exhaustion of both these resources, real memory free has a gives a lower time to exhaustion than used swap space. This is because of the difference in the initial and maximum/minimum values of these resources. Similar comparisons of slope and estimated time to exhaustion can be done on other machines. Overall, we find that the two resources file table size and process table size are not as important as used swap space and real memory free since they have a very small slope and high estimated times to failure due to exhaustion. Based on such comparisons, we can identify important and interesting resources to monitor and manage, to deal with aging related software failures.

The estimated time to resource exhaustion can be taken to be the estimated time of failure of the machine due to that particular resource. It is important to note that this only considers failure due to aging of a particular resource alone. For a more general failure prediction, the occurrence of transients in resource usage, as well as the combined effect of aging, periodic component and transient component, need to be modeled and understood. Additionally, the interaction and correlation between the usage of various resources and their impact on system availability remains to be explored. It is precisely for these reasons that the estimated times of failures done this way do not fully explain the actual times of failure observed on various machines. The estimated time to resource exhaustion and slope however can be used to study the aging phenomena which is a very important element in software failures. Prediction methods based on aging become important, especially in light of fault-tolerance techniques such as software rejuvenation where the time of actual rejuvenation is an issue.

\section{Conclusion}

In this paper, we described the design and implementation of a distributed monitoring and data collection tool. 
Based on SNMP, the tool is inter-operable among machines running UNIX and its variants. We also described the data collection process accomplished via monitoring operating system resource usage and system activity. The primary contribution of our work is a methodology for detecting and estimating aging in operational software. Based on this, we proposed a metric, "Estimated time to exhaustion", for each resource as a quantification of aging. The higher this metric is, lower is the effect of aging on this resource. This metric helps in comparing the effect of aging on different system resources and also in the identification of important resources to monitor and manage. This is also a first step towards predicting aging related failure occurrences, and may help us in developing a strategy for software fault-tolerance approaches, such as software rejuvenation, triggered by actual measurements.

\section{References}

[1] G. E. P. Box, G. M. Jenkins, and G. C. Reinsel. Time Series Analysis: Forecasting and Control. Prentice Hall, Englewood Cliffs, NJ, 1994.

[2] J.D. Case, M. Fedor, M.L. Schoffstall, C. Davin, M. T. Rose, and K. McCloghrie. Simple Network Management Protocol. RFC 1157, May 1990.

[3] R. Chillarege, S. Biyani, and J. Rosenthal. Measurement of failure rate in widely distributed software. In Proc. of 25 th IEEE Intl. Symposium on Fault-Tolerant Computing, pages 424-433, Pasadena, CA, July 1995.

[4] W. S. Cleveland. Robust locally weighted regression and smoothing scatterplots. Journal of the American Statistical Association, 74(368):829-836, December 1979.

[5] R. O. Gilbert. Statistical Methods for Environmental Pollution Monitoring. Van Nostrand Reinhold, New York, NY, 1987.

[6] J. Gray and D. P. Siewiorek. High-availability computer systems. IEEE Computer, pages 39-48, September 1991.

[7] J.P. Hansen and D.P. Siewiorek. Models for time coalescence in event logs. In Proc. of 22nd IEEE Intl. Symposium on FaultTolerant Computing, pages 221-227, 1992.

[8] Y. Huang, P. Jalote, and C. Kintala. Lecture Notes in Computer Science, Vol. 774, Two techniques for transient software error recovery, pages 159-170. Springer Verlag, Berlin, 1994.

[9] Y. Huang, C. Kintala, N. Kolettis, and N. D. Fulton. Software rejuvenation: Analysis, module and applications. In Proc. of 25th IEEE Intl. Symposium on Fault-Tolerant Computing, pages 381-390, Pasadena, California, June 1995.

[10] R.K. Iyer and D.J. Rossetti. Effect of system workload on operating system reliability: a study on IBM 3081. IEEE Transactions on Software Engineering, SE-11(12):1438-1448, Dec. 1985.

[11] R. K. Iyer, L. T. Young, and P. V. K. Iyer. Automatic recognition of intermittent failures: An experimental study of field data. IEEE Transactions on Computers, 39(4):525-537, April 1990.
[12] P. Jalote, Y. Huang, and C. Kintala. A framework for understanding and handling transient software failures. In Proc. of 2nd ISSAT Intl. Conf. on Reliability and Quality in Design, Orlando, Florida, 1995.

[13] H. Langendorfer J. Schonwalder. Tcl extensions for network management applications. In Proc. of 3rd Tcl/Tk Workshop, Toronto (Canada), July 1995.

[14] I. Lee, R. K. Iyer, and A. Mehta. Identifying software problems using symptoms. In Proc. of 24th IEEE Intl. Symposium on Fault-Tolerant Computing, Toulouse, France, June 1994.

[15] T-T. Lin and D. P. Siewiorek. Error log analysis: Statistical modeling and heuristic trend analysis. IEEE Transactions on Reliability, 39(4):419-432, October 1990.

[16] R. A. Maxion and F. E. Feather. A case study of Ethernet anomalies in a distributed computing environment. IEEE Transactions on Reliability, 39(4), 1990.

[17] M. T. Rose and K. McCloghrie. Structure and Identification of Management Information for TCP/IP-based Internets. RFC 1155, May 1990.

[18] P. K. Sen. Estimates of the regression coefficient based on Kendall's tau. Journal of the American Statistical Association, 63:1379-1389, 1968.

[19] M. Sullivan and R. Chillarege. Software defects and their impact on system availability - a study of field failures in operating systems. In Proc. of 21st IEEE Intl. Symposium on Fault-Tolerant Computing, pages 2-9, 1991.

[20] D. Tang and R. K. Iyer. Dependability measurement modeling of a multicomputer system. IEEE Transactions on Computers, 42(1), January 1993.

[21] A. Thakur and R. K. Iyer. Analyze-NOW - an environment for collection and analysis of failures in a network of workstations. In Proc. of 7th IEEE Intl. Symposium on Software Reliability Engineering, pages 14-23, White Plains, NY, April 1996. 